\title{
Diaphragmatic Hydatid Cyst, A Rare Entity Simulating A Thoracic Localization (About 4 Observations)
}

Benjelloun Hanane, Ami Meryem*, Zaghba Nahid, Yassine Najiba

Respiratory Diseases Department, Ibn Rochd University Hospital Casablanca, Casablanca 20250, Morocco

DOI: $10.36347 /$ sjmcr.2021.v09i02.013

| Received: 22.01.2021 | Accepted: 06.02.2021 | Published: 23.02.2021

*Corresponding author: Ami Meryem

Abstract

Original Research Article

The hydatid cyst of the diaphragm is defined by the development of a hydatid cyst in the thickness of the diaphragmatic muscle. This exceptional location, even in endemic countries, represents only 0.6 to $1.5 \%$ of all hydatid localizations. It poses topographic diagnostic problems with the pulmonary and sub diaphragmatic seat despite the contribution of ultrasonography and CT scan. The treatment is mainly surgical. The prognosis is generally good apart from the risk of recurrence. We report four observations of diaphragmatic hydatid cyst collected at the Respiratory Diseases Department of the Ibn Rochd University Hospital of Casablanca. They were two women and two men. The average age was 46.5 years. All of our patients were of rural origin. One patient was already operated on for hepatic hydatic cyst 11 years ago. Surgery was recommended in all four patients, combined with medical treatment. Intraoperative exploration had found an isolated diaphragmatic hydatid cyst in four cases, fistulized in the bronchi in two cases and ruptured in the pleural cavity in one case. The evolution was good after adequate care.

Keywords: Chest CT scanner, Diaphragm, Hydatidosis, Muscle hydatidosis; Surgery.

Copyright (C) 2021 The Author(s): This is an open-access article distributed under the terms of the Creative Commons Attribution 4.0 International License (CC BY-NC 4.0) which permits unrestricted use, distribution, and reproduction in any medium for non-commercial use provided the original author and source are credited.

\section{INTRODUCTION}

Hydatidosis is an endemic disease caused by Echinococcus granulosus. It is a health problem common mainly in the Mediterranean countries. The most frequently involved organs are the liver and lungs [1]. The diaphragmatic hydatid cyst is rarely reported in the literature. The diaphragmatic localization of the hydatic disease and its complications can pose a diagnostic challenge or influence therapeutic options. The purpose of this article is to examine the results of imagery of this rare localization with a focus on the clinical implications, modes of revelation and complications that guide therapeutic modalities.

\section{Patients And Methods}

This is a retrospective study carried out at the Respiratory Diseases Department of the Ibn Rochd University Hospital of Casablanca over 4 years from 2015 to 2019. It included four patients presenting an isolated diaphragmatic hydatidosis, simple, complicated or associated with a thoracic, abdominal or thoracoabdominal localization. Patient data was collected from their medical files. We have taken into account their epidemiological, clinical, radiological, therapeutic and evolutionary aspects.

\section{RESULTS}

Observation 1

Mr A.T. aged 18, without any particular pathological history, which had right basithoracic pain for 10 days, purulent bronchial syndrome, low abundance hemoptysis and effort dyspnea, without hydatidoptysis evolving in a context of febrile sensations and conservation of the general condition. The clinical examination had found a right posterobasal fluid effusion syndrome. The chest X-ray had shown a right paracardiac hydro-aeric image of thoracoabdominal seat performing the classic iceberg image (Fig-1). At the thoracic-abdominal CT, a diaphragmatic cystic image was found, measuring $95 / 85 \mathrm{~cm}$, with a hydro-aeric level within it (Fig-2). The assessment of the hydatid disease had not found any other intra- or extra-abdominal localizations. Hydatic serology was positive at $1 / 860$ in indirect hemagglutination (IHA). The bronchoscopy was normal. A right posterolateral thoracotomy in the 6th intercostal space (EIC) was performed, the exploration had revealed a multivesicular diaphragmatic cystic cavity with bronchial fistulas by lung invasion. An evacuation of the proliferative membranes intimately adhering to the pulmonary parenchyma was performed, as well as adhesiolysis revealing a diaphragmatic 
Benjelloun Hanane et al., Sch J Med Case Rep, Feb, 2021; 9(2): 167-171

connection of less than $1 \mathrm{~cm}$ having been aspirated and sutured. The patient was discharged from the hospital on the 7th postoperative day with antiparasitic treatment, after being made aware of preventive gestures. After a 12-month decline, the checkup had not been subject to thoracic or extra-thoracic hydatic recurrence.

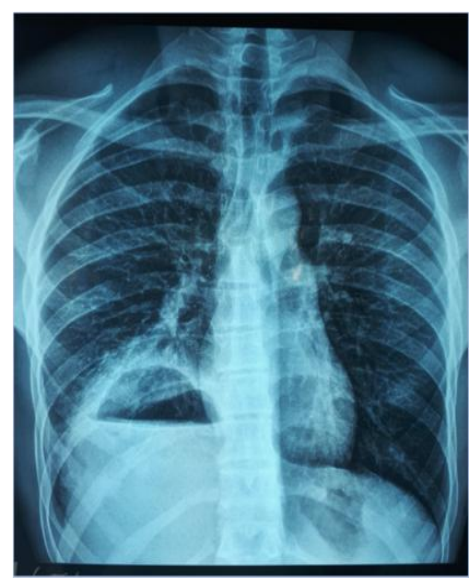

Fig-1: Chest $x$-ray showing a right paracardiac hydro-aeric image «iceberg image»

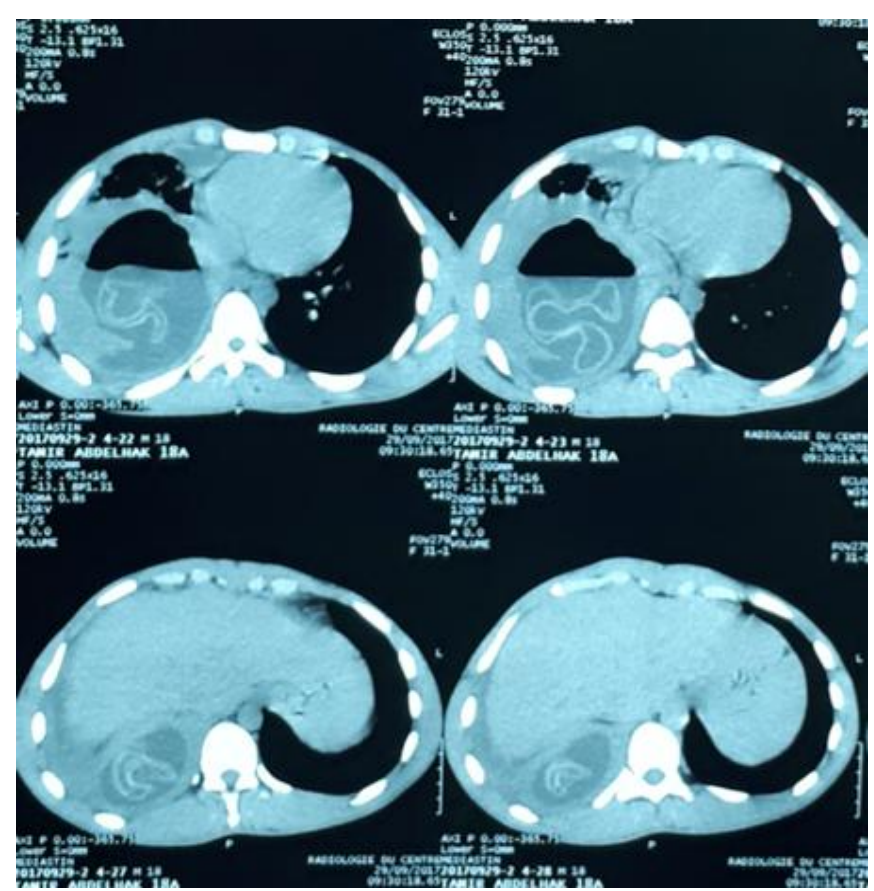

Fig-2: Thoraco-abdominal scanner showing a diaphragmatic cystic image measuring $95 / 85 \mathrm{~cm}$, with an hydro- aeric level and floating membranes

\section{Observation 2}

Ms. N.H, 59 years old, with no specific pathological history, had consulted for low abundance hemoptysis, hydatydoptysis and right basithoracic pain. The general condition was good and the clinical examination had found a right basithoracic matity and a sensitivity of the right hypochondrium. The chest X-ray had objectified an aspect of ascent of the right diaphragmatic dome. Hydatic serology was positive at $1 / 1320$ in IHA, and there were no extra thoracic localizations. The thoracic-abdominal scanner had shown a cystic image of the multivesicular hepatic dome, seat of calcifications, and measuring 110/90 mm with a condensation of right lower lobar pulmonary (Fig-3). Bronchial fibroscopy had objectified a thickening of the middle lobar spur without fistula or visible hydatic membranes. The patient had undergone a thoracoscopy converted to an anterior mini thoracotomy through the 4th right EIC on the anterior axillary line. On intraoperative exploration, the liver was normal, but the visualization of a diaphragmatic hydatid cyst was surprising. A fistula was found between the protruding dome of the diaphragmatic cyst and the lower lobe of the right lung. Protection of the lung parenchyma was done before performing a cystectomy and evacuation of the protruding membranes with fistula closure. Two aspirated Redon drains were placed in the residual diaphragmatic cavity. The postoperative follow-up was simple. An antihelminthic treatment was prescribed. 
Benjelloun Hanane et al., Sch J Med Case Rep, Feb, 2021; 9(2): 167-171
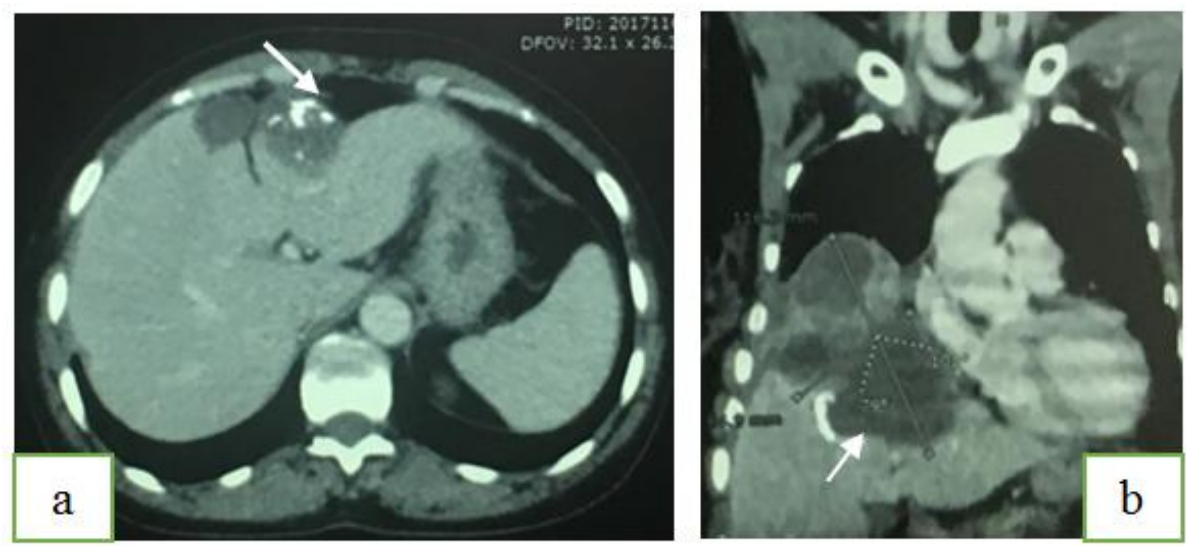

Fig-3: Thoraco-abdominal CT (a: cross section, b: sagittal section) showing a cystic image of the hepatic dome measuring $110 / 90 \mathrm{~mm}$ multivesicular seat of calcifications (arrows)

\section{Observation 3}

Mrs. R.A, 61 years old, of rural origin, was operated 11 years ago for a hydatid liver cyst. She had presented for one year a purulent bronchial syndrome complicated by several episodes of medium-abundant hemoptysis, without hydatidoptysis or rejection of membranes, associated with right basithoracic pain and dyspnea. All of this in a context of conservation of the general condition. The chest X-ray had shown right basal opacity posing a problem of its seat (supradiaphragmatic, diaphragmatic or sub-diaphragmatic) (Fig-4). The chest CT scan showed a multivesicular cystic image resting on the diaphragm with endothoracic development (Fig-4). Abdominal ultrasonography had found a dysmorphic liver without hydatid cyst. Hydatid serology and radiological exams for other hydatid localizations were negative. Endoscopic exploration had shown active bleeding from the right lower lobar bronchus, without visualization of hydatid membranes. The search for scolex in the bronchial aspiration fluid was negative. The patient had an iterative right posterolateral thoracotomy through the 7 th coast which was resected. Exploration had found at the right phrenic center of the diaphragmatic muscle, a multivesicular $\mathrm{KH}$ adhering to the base of the right lung. The surgical procedure consisted of adhesiolysis of the phrenic hydatid cyst and removal of the peri cyst after protected aspiration of the hydatic content. The patient was discharged from the hospital on the 5th postoperative day with antihelmintic treatment. the evolution was good.
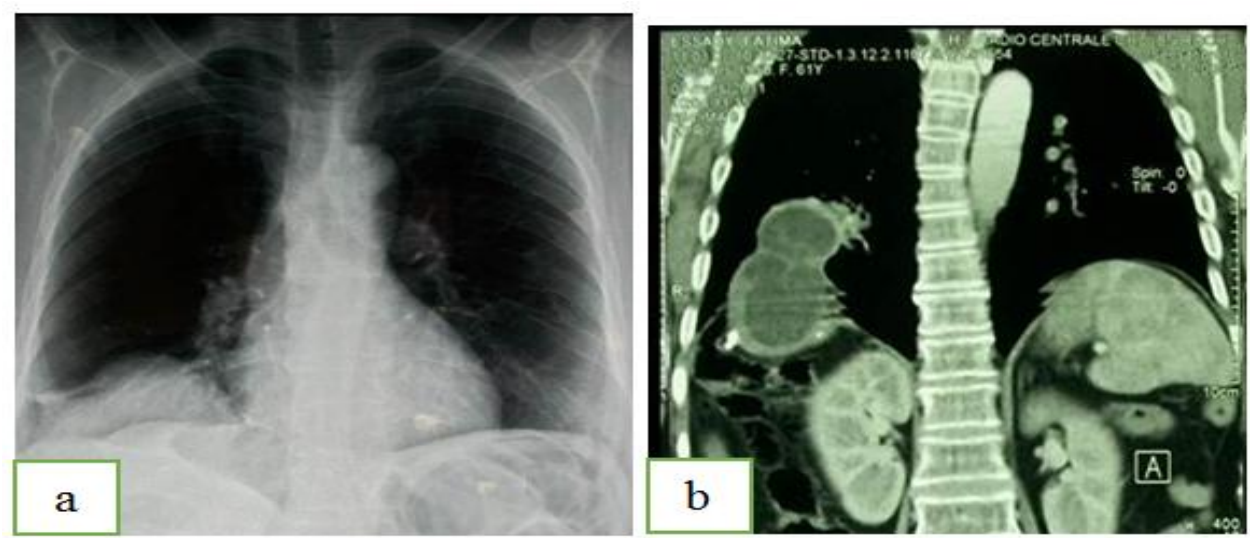

Fig-4: a. Chest x-ray showing a right basal opacity posing a problem of its seat. b. Chest scanner showing a hydatid cyst resting on the diaphragm with endothoracic development

\section{Observation 4}

Mr. M.G, aged 48, chronic smoker, with no other particular pathological history who complained for 2 months of a cough with purulent sputum, chest pain and dyspnea. The clinical examination had found a mixed effusion syndrome of the right hemithorax and a decline in the general condition. The chest X-ray had shown a right hydro-aeric image and the thoracic CT could not specify the pleural or parenchymal seat of this image (Fig-5). The bronchoscopy was normal. The intervention consisted of a posterolateral thoracotomy through the 6th right EIC. After opening the very thickened parietal pleura, exploration had shown the outcome of several liters of purulent liquid containing several hundred vesicles, some of which measured a few millimeters. At the diaphragm, an hydatic cyst was discovered with a fistula that released daughter blisters. Surgery consisted of debridement of the orifice, aspiration of the contents, and resection of the protruding dome. The pleural cavity was sterilized with hypertonic saline. The diaphragmatic orifice was sutured, then a pleural decortication allowing a good 


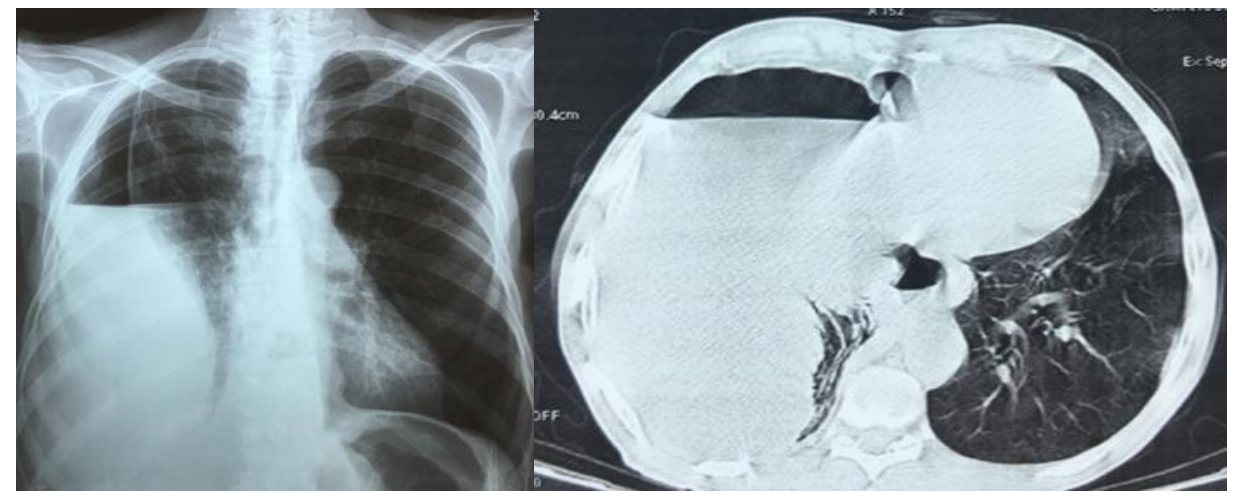

Fig-5: Chest $x$-ray and chest scanner showing a right hydro-aeric image

\section{DisCUSSION}

Hydatic disease, also called echinococcosis, is a cosmopolitan anthropozoonosis due to the presence and development in humans of the larval form of a taenia of the dog of the genus Echinococcus granulosus. It is rampant in the temperate grazing regions of the five continents where traditional pastoral farming takes place: The Mediterranean region including its southern shore, East Africa, Latin America, Australia and New Zealand. Incidence rates in these regions can exceed 50 cases per 100,000 person-years, with prevalence levels of up to $5 \%$ to $10 \%$ [2]. The four most reputable countries with hydatid endemicity are: Uruguay (32/100,000), Argentina $(21 / 100,000)$, Tunisia $(15 / 100,000)$, and Morocco $(7.2 / 100,000)$ [4].

The first localization of hydatidosis is hepatic $(50-63 \%)$. The muscle represents the 4th localization, after the liver, lung and spleen, and especially the diaphragmatic muscle. Diaphragmatic hydatidosis develops in the thickness of the diaphragmatic muscle between the pleural and peritoneal serous. It is one of the rarest extra-pulmonary thoracic localizations of hydatic cysts. It represents 0.6 to $1.5 \%[3,8]$. The rarity of this localization can be explained, on the one hand, by the contractility of the diaphragmatic muscle preventing the fixation of the parasite and, on the other hand, by the production of lactic acid which often prevents the development of the embryo in the muscle [4]. Diaphragmatic involvement may be primary through blood or lymphatic pathways, isolated or associated with other locations. This primitive form is attested by the absence of a history of peridiaphragmatic hydatic cyst surgery [4]. It was found in three of our patients (observation 1,2,4). the second patient (observation 2), had a rupture of the diaphragmatic hydatid cyst in the lung. This rupture occurs in the event of significant expansion or infection weakening the wall of the cyst, as well as the friction of the peri cyst with the pulmonary parenchyma [5].

Common signs of hydatid pulmonary disease include chest pain, hemoptysis, hydatidoptysis and symptoms of pneumonia [6]. Bronchial endoscopy could contribute to the diagnosis by showing a fistula containing a hydatic membrane. It is indicated in the presence of hemoptysis in order to eliminate a tumor pathology [7]. The fourth patient (observation 4), had presented a rupture of the cyst in the pleural cavity. It was a hydatid pyopneumothorax following spontaneous rupture of a diaphragmatic cyst. This is a very rare situation that has been described by only a few authors $[8,9]$. It would be due, on the one hand, to an exaggerated tension of the cyst due to its size or the constraint to its expansion exerted by the diaphragmatic muscle. On the other hand, the negative pressure prevailing in the pleural cavity would accentuate the cracking of this cyst under tension, leading to its rupture. The clinical picture is that of a pyopneumothorax associating a productive cough, dyspnea, fever, chest pain, and altered general condition. X-rays would allow us to discuss the different differential diagnostics of pyopneumothorax, in particular the tubercular origin in our endemic context.

Ultrasound, CT and magnetic resonance imaging (MRI) can be used in conjunction with chest $\mathrm{x}$ ray to detect the location of the hydatic cyst. However, it becomes more complicated when it is perforated. Kabiri et al, reported an $82 \%$ inaccurate diagnostic percentage when abdominal ultrasound and thoracic CT were used in a study of 27 diaphragmatic hydatic cysts [8]. Therefore, most diaphragmatic cysts are diagnosed in operation. For example, and like our 2nd case, many patients benefited from preoperative radiological investigations in favor of hepatic or pulmonary hydatic localization, while surgical exploration revealed hydatid cysts with a diaphragmatic seat.

As for the secondary forms, they are caused by a peritoneal seeding from a liver cyst in general [10]. This secondary form was found in the 3rd observation. It would be the consequence of contamination by an open cyst near the diaphragm after surgery or spontaneous rupture. 
Benjelloun Hanane et al., Sch J Med Case Rep, Feb, 2021; 9(2): 167-171

Various serological tests are available for the diagnosis, screening and postoperative follow-up of recurrences. These are hydatic immunoelectrophoresis, immunoenzymatic assay, latex agglutination and the IHA test [11]. Hydatic serology is rarely positive when the hydatid cyst is isolated, and may remain negative after the intervention, even in case of recurrence as in the case of our 3rd patient [8]. However, a negative test does not exclude echinococcosis [12].

Surgery should be the treatment of choice for hydatic disease. In KHD, excision of the cyst by thoracotomy is an appropriate approach [13]. If combined with liver localization, these cysts can be treated simultaneously in a single step. If the cyst ruptures in the pleura, cystostomy or cystectomy and dimpling (obliteration of the cavity by suturing the cavity walls) are commonly practiced with or without pleural decortication $[14,15]$. The bronchopleural fistula can be identified intraoperatively and then repaired [14]. Lung resections (segmentectomy or lobectomy) are occasionally performed, when the pulmonary parenchyma adjacent to the cyst is destroyed, unable to expand, or both [15]. A medical treatment based on albendazole should be systematically prescribed in diaphragmatic hydatid cysts because of their multivesiculation rich in protoscolex. Some authors offer a bi-therapy combining praziquantel. Indeed, the indications for medical treatment remain the multifocal, multivesicular and preoperative hydatid cyst to reduce protoscolex and the risk of secondary hydatidosis and recurrence [16]. Breaking the parasitic cycle remains the best preventive treatment, based on hygiene measures, health education for populations, and the fight against stray dogs.

\section{CONCLUSiON}

The diaphragmatic localization of hydatid cyst is rare and does not seem to be of any particular gravity. The modes of revelation can be multiple. If the diagnosis of the hydatic nature does not pose any particular problem and is based on the ultrasound examination, the confirmation of the diaphragmatic seat requires the use of more efficient imaging techniques, and can only be certain in operation. The prognosis is generally good apart from the risk of recurrence. Medical treatment with albendazole could improve this risk and thus ensure good long-term development. We insist on the importance of primary prevention, in particular hand hygiene, which constitutes an important pillar in the management of hydatidosis.

\section{BIBLIOGRAPHY}

1. Echinococcose. Available from https://www.who.int/fr/news-room/factsheets/detail/echinococcosis.
2. Makni A, Fetirich F, Jouini M, Kacem M, Ben Safta Z. Kyste hydatique primitif et isolé du diaphragme en Tunisie. Bull Soc Pathol Exot. 2012; 105:262-4.

3. Guedj P, Atsamena M, Lagoutine V. Kyste hydatique primitif du diaphragme ouvert dans les voies bronchiques. Ann Chir Thorac Cardiovasc. 1973; 12:175-9.

4. Daali M, Hssaida R. Kyste hydatique du diaphragme rompu dans la plèvre : à propos d'un cas. Ann Chir. 2000;125:493-5.

5. Baram A, Kakamad FH, Alwan AA. Primary posterior mediastinal hydatid cyst mimicking malignant mediastinal neurogenic tumor. Int J Case Rep. 2014; 5:54-57.

6. Saimato AG. Medical treatment of liver hydatidosis. World J. Surg. 2001; 25: 15-2.

7. Issoufou I, Harmouchi $\mathrm{H}$, Rabiou S, Belliraj L, Ammor FZ, Diarra AS, Lakranbi M, Sani R, Ouadnouni Y, Smahi M. La chirurgie des hydatidoses diaphragmatiques et leurs complications. Revue de Pneumologie Clinique. 2017 Oct 1;73(5):253-7.

8. Kabiri H, Al Aziz S, El Maslout A, Benosman AC. L'hydatidose diaphragmatique. Rev Pneumol Clin. 2000; 57:13-9.

9. Racil H, Amar JB, Moulay RE, Ridene I, Cheikrouhou S, Zarrouk M, Chaouch N, Chabbou A. Kystes hydatiques compliqués du poumon. Revue des maladies respiratoires. 2009 Sep 1;26(7):727-34.

10. Bouchikh M, Achira A, Maidia M, Ouchen F, Fenane H, Benosman A. La rupture intrapleurale des kystes hydatiques pulmonaires. Rev Pneumol clin. 2014; 70:203-7.

11. Zarzosa MP, Orduna D, Gutierrez P. Evaluation of six serological tests in diagnosis and postoperative control of pulmonary hydatid disease patients. Microbiol Infect Dis. 1999; 35:255-262.

12. Abu-Eshy SA. Some rare presentations of hydatid cyst (Echinococcus granulosus). J R Coll Surg Edinb. 1998; 43:347-352.

13. Eren S, Ulku R, Tanrikulu AC, Eren MN. Primary giant hydatid cyst of the diaphragm. Ann Thorac Cardiovasc Surg. 2004; 10:118-119.

14. Aribas OK, Kanat F, Gormus N, Turk E. Complications pleurales de la maladie hydatique. J Thorac Cardiovasc Surg. 2002; 123:492-497.

15. Puri D, Mandal AK, Kaur HP, Mahant TS. Kyste hydatique rompu avec une présentation inhabituelle. Case Rep Surg. 2011; 730604.

16. Stamatakos M, Sargedi C, Stefanaki C, Safioleas C, Matthaiopoulou I, Safioleas M. Anthelminthic treatment: an adjuvant therapeutic strategy against Echinococcus granulosus. Parasitology international. 2009 Jun 1;58(2):115-20. 